\title{
Prediction of Surface Subsidence Caused by Rectangular Pipe Jacking Construction
}

\author{
Chao ZHANG ${ }^{1}$, Qi Jun JIA ${ }^{1}$, Ri Qing XU², Su Yang FENG ${ }^{2 *}$ \\ ${ }^{1}$ China Road and Bridge Corporation, Dongcheng, Beijing 100011, China \\ ${ }^{2}$ College of Civil Engineering and Architecture, Zhejiang University, Hangzhou, Zhejiang 310058, China
}

\begin{abstract}
Based on the development of rectangular pipe jacking, the characteristics of rectangular pipe jacking are introduced. The difference between surface settlements caused by rectangular pipe jacking is calculated by stochastic medium method and peck formula method. The influence of section shape on rectangular pipe jacking is analyzed. The results show that the calculation results of the stochastic medium method are more reasonable.
\end{abstract}

\section{Introduction}

Pipe jacking is a technique used for installing pipes under the ground using a tunnel boring machine and hydraulic jacks located inside a jacking pit. A shield is inserted into the ground through a drive shaft or reception point and cuts a bore. As the shield advances forward, excavating the earth in its path, sections of pipe are jacked into place directly behind it using powerful hydraulic jacks. As a means of trenchless construction, the pipe jacking technology has been fully developed in the world. It has accumulated a lot of experience in terms of the large diameter, long distance and curve jacking construction [1-3].

The cross-section of the jacking is mostly circular. In the early 1970s, the rectangular pipe jacking technology was used in Japan for the first time. While in 1999, the first rectangular pipe jacking was completed in the underground pavement project of entrance 5\# in Lujiazui station, line 2, Shanghai Metro, China[4].

Since most rectangular pipe jacking projects are located in the bustling area, there is a high demand for surface subsidence control. The existing calculation methods of ground settlement include Peck formula method [5] and stochastic medium method [6] [7]. Peck formula method can be applied to circular tunnel and jacking, but the rectangular pipe jacking remains to be verified. Nevertheless, stochastic medium method can be used for a variety of shapes of tunnels or pipe jacking. Wang [7] calculated the surface subsidence induced by ground loss under rectangular pipe jacking construction. However, it was assumed that the soil in the rectangular pipe jacking was uniform convergence, and lacked in-depth study. In this paper, stochastic medium method is used to calculate the surface subsidence caused by rectangular pipe jacking under uniform and inhomogeneous convergence modes. What's more, compared with measured values, the applicability of Peck formula in rectangular pipe jacking is discussed.

\section{The characteristics of rectangular pipe jacking}

This paper collects some representative pipe jacking projects around the world. As can be seen from Table 1, the cross-section of the rectangular pipe jacking is too large, as the equivalent radius is often greater than $1.8 \mathrm{~m}$ and it can be regarded as large diameter pipe jacking according to the code for circular one. It also indicates that the depth is shallow because the ratio of depth to the equivalent radius is usually less than 3 . Besides, the jacking path is shorter. Rectangular pipe jacking is often used for subway entrances; the ground outside is mostly trunk lines and dense buildings, so a stricter ground subsidence control standard is required.

Table 1 Partial geometric parameters of rectangular pipe jacking projects

\begin{tabular}{cccccc}
\hline Project & $\begin{array}{c}\text { Buried } \\
\text { Depth /m }\end{array}$ & Width $\times$ height & $\mathbf{z} / \boldsymbol{R}^{\prime}$ & $\begin{array}{c}\text { Jacking } \\
\text { Path /m }\end{array}$ & $\begin{array}{c}\text { Number of } \\
\text { Jacking }\end{array}$ \\
\hline $\begin{array}{c}\text { Underpass tunnel in Zhongzhou } \\
\text { Avenue, Zhengzhou, China }\end{array}$ & 8 & $9.0 \mathrm{~m} \times 6.1 \mathrm{~m}$ & 1.91 & 110 & 4 \\
Entrance 5\# in Culture Park Station, & 6.9 & $6 \mathrm{~m} \times 4.3 \mathrm{~m}$ & 2.41 & 52 & 1 \\
\hline
\end{tabular}

\footnotetext{
* Corresponding author: fsy@zju.edu.cn
} 


\begin{tabular}{|c|c|c|c|c|c|}
\hline line 6, Guangzhou Metro, China & & & & & \\
\hline $\begin{array}{l}\text { Entrance } 4 \# \text { in Wangjiadun Station, } \\
\text { line 2, Wuhan Metro, China }\end{array}$ & 7.9 & $6 \mathrm{~m} \times 4 \mathrm{~m}$ & 2.86 & 62 & 1 \\
\hline $\begin{array}{c}\text { Entrance } 3 \# \text { in Pudian Road Station, } \\
\text { line } 6 \text {, Shanghai Metro, China }\end{array}$ & 7.9 & $6.2 \mathrm{~m} \times 4.4 \mathrm{~m}$ & 2.68 & 42 & 1 \\
\hline $\begin{array}{l}\text { Channel from Huaqiang North Station } \\
\text { to Huaxin Station, Shenzhen, China }\end{array}$ & 5.95 & $6.9 \mathrm{~m} \times 4.9 \mathrm{~m}$ & 1.81 & 41 & 3 \\
\hline $\begin{array}{l}\text { Sewer tunnel in Kikuta-gawaroute 2, } \\
\text { Narashino, Chiba-ken, Japan }\end{array}$ & 5.0 & $4.4 \mathrm{~m} \times 4.0 \mathrm{~m}$ & 2.11 & & 2 \\
\hline $\begin{array}{c}\text { Box-type pipe jacking pedestrian } \\
\text { tunnel in Kota, Indonesia }\end{array}$ & 6.65 & $6 \mathrm{~m} \times 4 \mathrm{~m}$ & 2.41 & 45 & 1 \\
\hline
\end{tabular}

\section{Stochastic medium theory}

The whole excavation is decomposed into infinitely small excavations from a statistical point of view, assuming that the soil is not condensed, the density does not change, and the rock mass is incompressible. The effect of the upper rock mass should be equal to the sum of the effects of infinitely small excavations. That is what we call stochastic medium theory. Under the plane strain condition, the ground traverse settlement trough caused by excavating the micro-unit at $\operatorname{deep} z$ is:

$$
\begin{gathered}
s_{e}(x)=\frac{1}{r(z)} \exp \left(-\frac{\pi}{r(z)^{2}} x^{2}\right) d \xi d \eta \\
r(z)=z / \tan \beta
\end{gathered}
$$

Where $r(z)$ is the main influence radius on the horizontal plane, otherwise known as the main scope of influence, $\beta$ is the main influencing angle of upper tunnel surrounding rock. The relationship between $\beta$ and the width of settlement trough $i$ can be obtained by comparing Peck formula method and stochastic medium method:

$$
\tan \beta=\frac{z_{0}}{\sqrt{2 \pi} i}
$$

The calculation method of $i$ value in stochastic medium method includes Knothe Formula and the method proposed by Han [8].

(1) Knothe Formula

$$
i=\frac{z_{0}}{\sqrt{2 \pi} \tan \left(45^{\circ}-\frac{\varphi}{2}\right)}
$$

Where $\varphi$ is internal friction angle of soil.

(2) Han's method

$$
\begin{gathered}
i=K z_{0} \\
\tan \beta=\frac{20}{50-\varphi}
\end{gathered}
$$

Where $K$ is based on the experiences of different types of soil.

As Knothe Formula is mainly applied to all types of rock, while pipe jacking technology is usually used in soft soil areas, the second method is used to calculate the influence angle $\beta$ in this paper.
The calculation of the surface settlement horizontally caused by ground loss can be obtained by integrating the settlement area:

$$
s(x)=\iint_{\Omega} \frac{1}{r(z)} \exp \left(-\frac{\pi}{r(z)^{2}} x^{2}\right) d \xi d \eta
$$

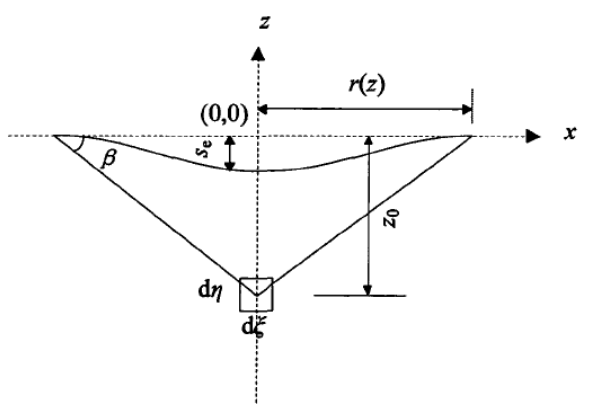

Figure 1 The excavation of unit under the plane strain condition

With reference to the engineering experiences of circular pipe jacking [8], there are two kinds of convergence modes for rectangular pipe jacking, uniform convergence and non-uniform convergence. In the uniform convergence mode, the pipe jack is suspended in the slurry jacket, and the gap between the top pipe and the soil is equal to the bottom clearance. In the non-uniform convergence mode, by contrast, the jacking is sinking as a whole and the bottom clearance is zero.

When tunneling machine advancing, the angle between the advancing direction and the horizontal direction is greater than zero during rectangular pipe jacking. And the cross section of rectangular pipe jacking is generally large; the bottom of the pipe is in direct contact with the soil due to its own weight. Therefore non-uniform convergence mode is more in line with the actual situation.

Assuming $\Delta A=k \Delta B, \Delta B$ can be inferred by the rate of ground loss:

$$
\Delta B=\frac{1}{4 k}\left[-(A+k B)+\sqrt{(A+k B)^{2}+4 \frac{k A B \varepsilon}{1-\varepsilon}}\right]
$$

Where $\varepsilon$ is the rate of ground loss, that is, the amount of ground loss is divided by the actual excavation area of the pipe jacking. 


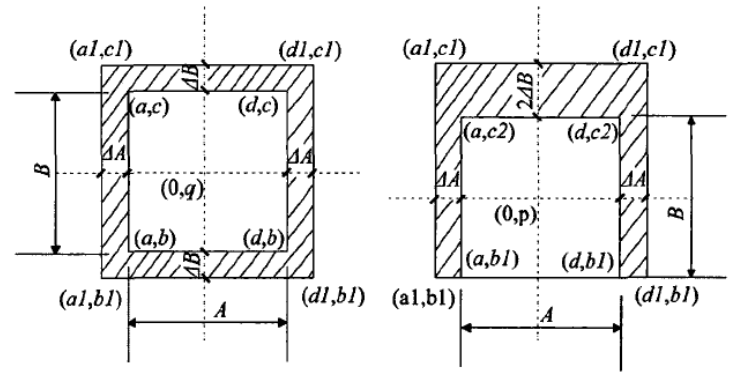

Figure 2 Uniform and non-uniform convergence mode

Take the vertical axis of the surface $z=0$, the be obtained:

calculation formula of soil settlement in two modes can (1) Uniform convergence mode

$$
s(x)=\int_{b 1}^{c 1} \int_{a 1}^{d 1} \frac{\tan \beta}{\eta} \exp \left[-\frac{\pi \tan ^{2} \beta}{\eta^{2}}(x-\xi)^{2}\right] \mathrm{d} \xi \mathrm{d} \eta-\int_{b}^{c} \int_{a}^{d} \frac{\tan \beta}{\eta} \exp \left[-\frac{\pi \tan ^{2} \beta}{\eta^{2}}(x-\xi)^{2}\right] \mathrm{d} \xi \mathrm{d} \eta
$$

(2) Non-uniform convergence mode

$$
\begin{gathered}
s(x)=\int_{b 1}^{c 1} \int_{a 1}^{d 1} \frac{\tan \beta}{\eta} \exp \left[-\frac{\pi \tan ^{2} \beta}{\eta^{2}}(x-\xi)^{2}\right] \mathrm{d} \xi \mathrm{d} \eta-\int_{b 1}^{c 2} \int_{a}^{d} \frac{\tan \beta}{\eta} \exp \left[-\frac{\pi \tan ^{2} \beta}{\eta^{2}}(x-\xi)^{2}\right] \mathrm{d} \xi \mathrm{d} \eta \\
\text { non-uniform convergence }
\end{gathered}
$$

\section{Comparison of stochastic medium method and Peck formula method}

In order to estimate the settlement, Peck put forward the empirical formula for the shield tunnel or circular pipe jacking, that is, Peck formula.

$$
\begin{gathered}
S(x)=S_{\max } e^{-\frac{x^{2}}{2 i^{2}}} \\
S_{\max }=\frac{V_{\text {loss }}}{i \sqrt{2 \pi}}
\end{gathered}
$$

Where $S(x)$ is the amount of ground subsidence, $x$ is the transverse horizontal distance from the axis of the tunnel, $S_{\max }$ is the maximum settlement above the tunnel axis, $V_{\text {loss }}$ is the amount of ground loss per unit length of the tunnel, $i$ is the width of settlement trough.

Peck formula was summarized on the basis of a large number of measured data, most of that was deep circular tunnel [9], while rectangle pipe jacking was mainly shallow buried. As a result, the accuracy of Peck in predicting ground settlement trough of a rectangular pipe is still to be studied. In order to compare the differences between the stochastic medium method and Peck formula, the two types of methods are used to calculate the surface subsidence of rectangular pipe jacking under different burial conditions. Supposing that $A=5 \mathrm{~m}, B=5 \mathrm{~m}$, the top clearance $\Delta A$ is equal to the bottom clearance $\Delta B$, and the rate of ground loss is $1 \%$. In addition, the stochastic medium method is divided into two modes: uniform convergence and non-uniform convergence.
As shown in the figure, there is a big difference between the uniform convergence mode and the non-uniform convergence mode. The maximum settlement value of the uniform convergence mode is always smaller than that of the non-uniform convergence mode. The latter settlement trough is narrow and deep, while the former one is wide and shallow. As the burial depth decreases, the difference between the two modes is increasing.

With respect to the uniform convergence mode, the bottom clearance is moved to the top in the non-uniform convergence mode. This shows the influence of the location of ground loss on surface subsidence. The amount of ground loss in the same condition, the more shallow the location of the loss, the greater the impact on the surface.

(2) The differences between stochastic medium method and Peck formula

Han [10] theorized that if the depth was deep enough or the excavation unit was small enough, the results of the two methods should be consistent. To verify his theory, two methods were used to calculate the settlement of the ground caused by circular tunnel construction. It turned out that the calculated results of Peck formula were between the results of the uniform and non-uniform convergence modes when using the stochastic medium method. With the increase of the buried depth, the gap between the three decreased gradually, but not completely consistent.

(1) Comparison of uniform convergence and

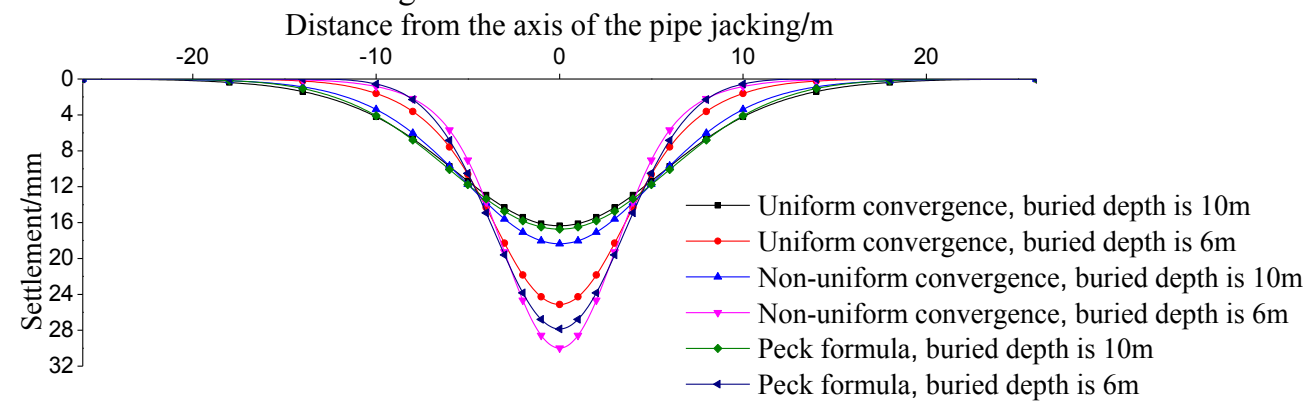

Figure 3 Comparison of stochastic medium method and Peck formula under shallow buried condition 


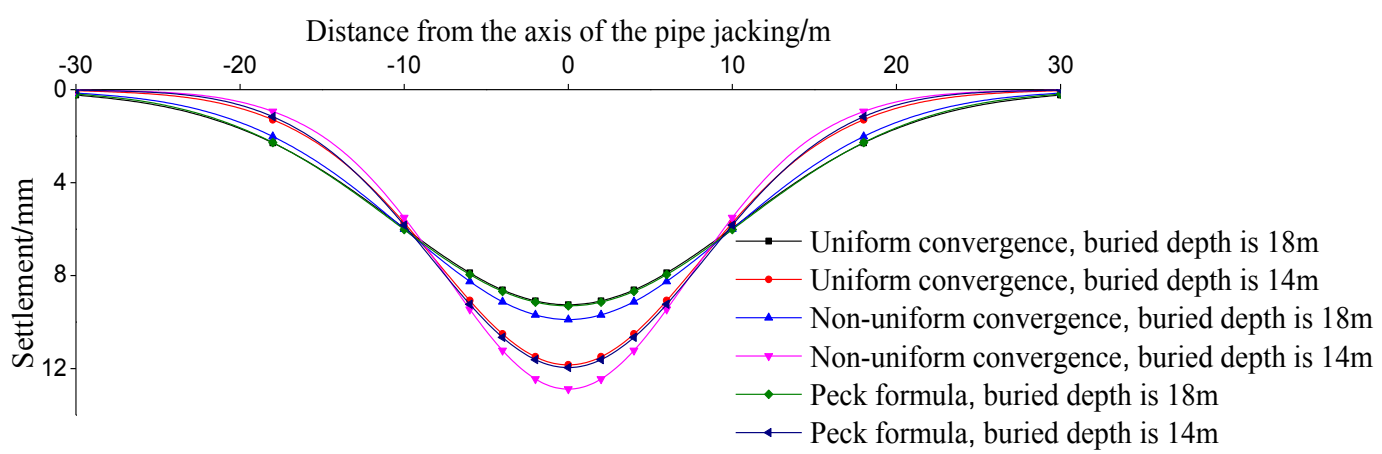

Figure 4 Comparison of stochastic medium method and Peck formula under deep buried condition

As shown in Fig. 3 and Fig. 4, the calculated results are consistent with those of Han's [10].The calculated results of Peck formula were between the results of the uniform and non-uniform convergence modes when using the stochastic medium method. With the increase of the buried depth, the results of the uniform model are consistent with those of Peck formula. However, there are always some differences between the calculation results of the non-uniform convergence model and those of Peck formula. It demonstrates that Peck formula is more suitable for predicting the subsidence of deep buried tunnels under uniform convergence mode. For shallow buried pipes, the results when using Peck formula to predict and actual results often are put in certain error.
(1) Comparison of round and rectangular pipe jacking

In order to compare the differences between the circular and the rectangular pipe jacking, a circular pipe jacking model is established to calculate the surface subsidence under non-uniform convergence conditions. The pipe jacking area and the stratum loss rate are set the same as the previous example.

Compared with the calculation results of the two types of pipe jacking, the shape of the two settlement trough is not very different on the condition that both have the same height and width. When the depth is shallow, the maximum settlement value of circular pipe is slightly smaller than the rectangular one. While when the depth is deep, the settlement regularities of circular and rectangular pipe are basically the same.

\section{The effect of the cross-sectional shape}

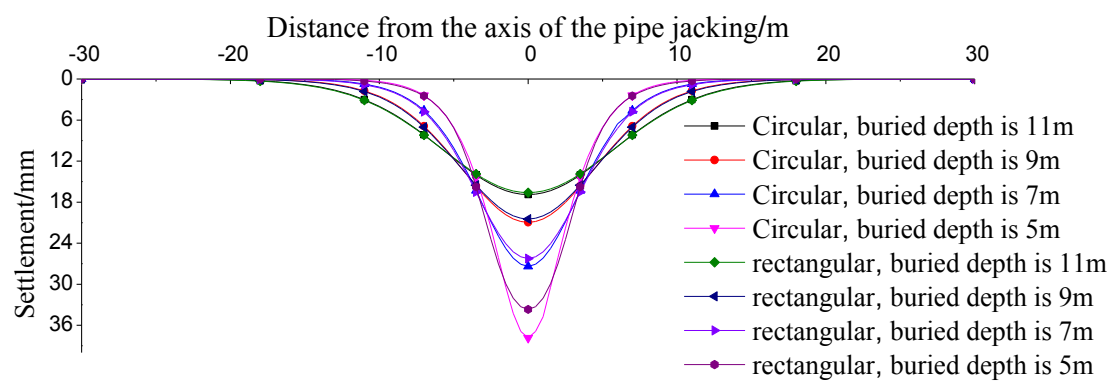

Figure 5 Comparison of surface subsidence between rectangular and circular pipe jacking under different buried depth

(2) The influence of pipe width on surface subsidence

Due to the particularity of rectangular pipe jacking, the width of the rectangular pipe jacking is often greater than the height; the aspect ratio is up to 2.0 in actual projects. In order to study the influence of the aspect ratio on the surface subsidence, this paper changes the width of the pipe to obtain a different aspect ratio. Meanwhile, the height and the stratum loss rate are consistent with the previous example, and the depth of the pipe is set to $-8 \mathrm{~m}$. The results are shown in Fig. 6 .

Distance from the axis of the pipe jacking $/ \mathrm{m}$

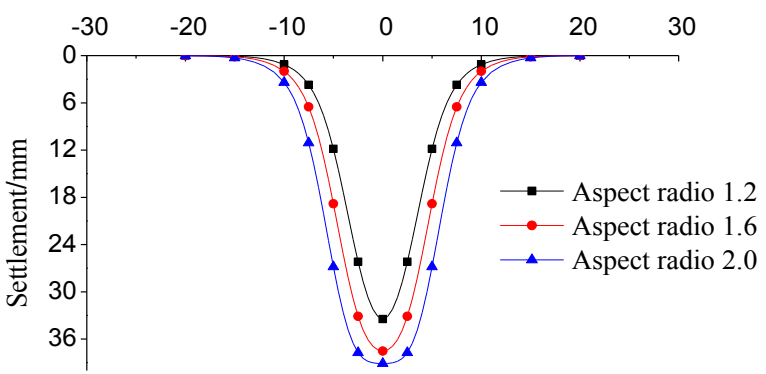

Figure 6 Surface subsidence curves under different aspect ratios

With the increase of the aspect ratio, the actual excavation area increases and the total loss of stratum increases correspondingly. Moreover, the settlement trough curve moves down as a whole, and the width of settlement trough also increases. Take the settlement of $18 \mathrm{~mm}$ corresponding to the abscissa $i_{18}$, and the width of 
settlement trough is quantitatively analyzed with $i_{18}$ as the standard. As shown in Fig. 7 and Fig. 8, the width of settlement trough is linearly related to the aspect ratio, and the maximum settlement shows an exponential relation with the aspect ratio.

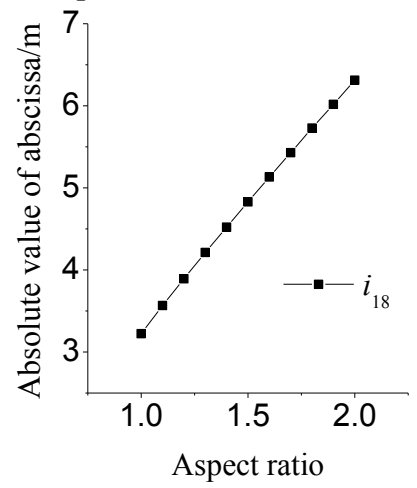

Figure 7 The relationship between $i_{18}$ and the aspect ratio

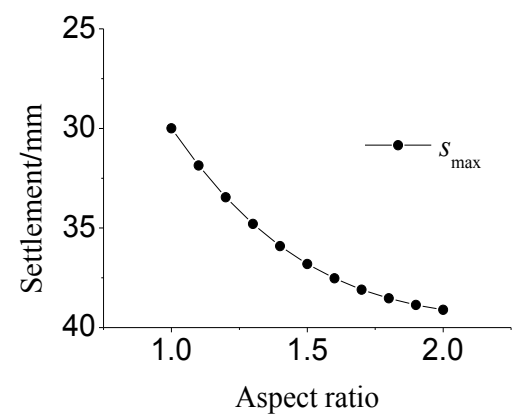

Figure 8 The relationship between the maximum settlement and the aspect ratio

\section{Influence of $k$}

According to the gap parameters proposed by Lee[11], the gap $g$ between the soil and the pipe jacking is mainly composed of three parts:

$$
g=G_{p}+U_{3 D}+\omega
$$

Where $G_{p}$ is the physical gap, usually refers to the difference between the external dimensions of the roadheader and the pipe; $U_{3 D}$ is the three-dimensional elastic-plastic deformation of the front part of the roadheader; $\omega$ is the construction factors (including shield steering adjustment, throwing, retreat, buckle, etc.). For the rectangular pipe jacking, the physical gap between the top and the bottom is greater than or equal to the physical gap on both sides, such as channel from Shenzhen Huaqiang North Station to Huaxin Station, the physical gap on both sides is one-tenth that between the top and the bottom. And due to the existence of hydrostatic pressure and lateral earth pressure, the mud supporting role on both sides is greater than the top. Therefore, the ratio $k$ of $\Delta A$ to $\Delta B$ should be less than 1 .

In this paper, the influence of the physical gap upon the surface subsidence on both sides is studied by changing the ratio of $\Delta A$ to $\Delta B$. Meanwhile, the size of pipe jacking and the amount of stratum loss are consistent with the previous example. The results are shown in Fig. 9. The smaller the proportion of stratum loss on both sides of the total, the deeper and the narrower the settlement trough is. What's more, the curve passes through $( \pm 3.5,-16.6)$ regardless of how the $k$ value changes. In other words, other things being equal, the settling curve always passes through the point where the settlement value is about half of the maximum settlement value.

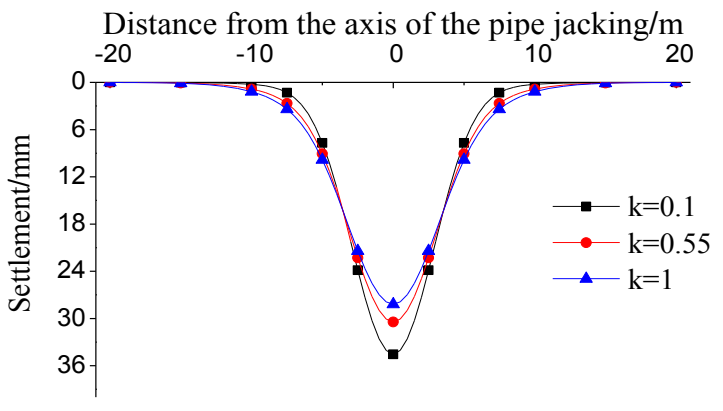

Figure 9 Influence of $k$ value on surface subsidence

\section{Example}

A slurry pressure balanced rectangular pipe jacking machine is used for construction in the exit of a subway station. The length of the pipe is $43.5 \mathrm{~m}$, the slope is $0.5 \%$ and the average covering thickness is $5.2 \mathrm{~m}$. The external dimension of the pipe is $6.0 \mathrm{~m} \times 4.2 \mathrm{~m}$. The surrounding soil is silt fine sand and silty clay, where the internal friction angle $\varphi$ ranges from $10^{\circ}$ to $16^{\circ}$.According to the literature and the data, we take $\tan \beta$ as 0.59.By calculating the clearance ratio $k=0.1$. In order to better compare with the measured values, suppose that the stratum loss rate $\varepsilon$ is $0.77 \%$ in Peck formula, $0.62 \%$ in the stochastic medium method, and the convergence mode is assumed to be non-uniform.

As shown in Fig. 10, Peck formula and the stochastic medium method can achieve good results. However, the curve obtained by the Peck formula is relatively wide. While the curve obtained by the stochastic medium method is closer to the measured value whose shape is narrow and deep. It is more reasonable to use the stochastic medium method to calculate the settlement of the rectangular pipe jacking. 


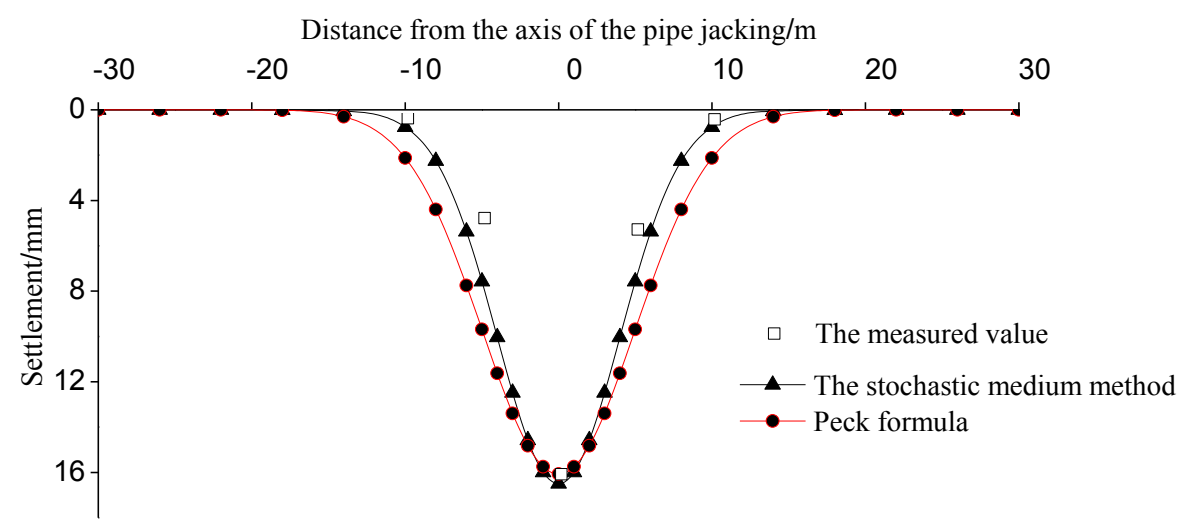

Figure 10 Comparison of the measured and calculated values

\section{Conclusions}

In this paper, the stochastic medium method is used to calculate the surface settlement caused by rectangular pipe jacking. Compared with the calculation results of Peck formula, the results show that:

(1) The maximum settlement of the uniform convergence mode is smaller than that of the non-uniform convergence mode. The settlement trough is wide and shallow under the uniform convergence mode, while narrow and deep under the non-uniform convergence mode. As the burial depth decreases, the difference between the two modes is increasing.

(2) The calculated results of Peck formula are between the results of the uniform and non-uniform convergence modes when using the stochastic medium method. With the increase of the buried depth, the results of the uniform model are consistent with those of Peck formula, while the results under the non-uniform convergence mode and those of Peck formula always have a certain difference.

(3) With the increase of the buried depth, the settlement trough of the circular and rectangular pipe jacking tends to be the same. The width of settlement trough increases linearly with increase of the aspect ratio.

(4) The increase in the clearance ratio of the settlement trough tends to be wide and shallow, but always through the fixed point.

(5) Compared with the measured results, the calculation results of the stochastic medium method are more reasonable.

\section{References}

1. ZHANG Yu-sheng, ZHOU Ming-yi. Jacking process of a pipe with large diameter through a long distance[J]. Chinese Journal of Geotechnical Engineering, 2000,04:506-508.

2. ZHU He-hua, WU Jiang-bin, PAN Tong-yan. Theoretical analysis of three-dimensional mechanical model of curved pipe jacking and its application[J]. Chinese Journal of Geotechnical Engineering, 2003,04:492-495.
3. WEI Gang, XU Ri-qing, HUANG Bin. Analysis of stability failure for pipeline during long distance pipe jacking $[\mathrm{J}]$. Chinese Journal of Rock Mechanics and Engineering, 2005,08:1427-1432.

4. LV Jian-zhong, YANG Lei. Jacking construction method for soil pressure balance stylerectangle jacking-pipe (YJGF15-2000)[J]. Construction Technology, 2002,09:43-45.

5. LIN Qiang-qiang. Analysis and control of the surface deformation caused by rectangular pipe jacking construction[D].Tongji University,2008.

6. WANG Ri-dong. Analysis of additional load imposed on underground pipelines nearby induced by the construction of rectangular pipejacking[J].Low Temperature Architecture Technology,2014,09:124-127.

7. WANG Ri-dong. Research of computing method of soil deformation induced by the construction of rectangular pipejacking[J]. Journal of Jamusi University, 2014,05:711-714+722.

8. Dingwen Zhang, Bo Liu, Yujun Qin. Construction of a large-section long pedestrian underpass using pipe jacking in muddy silty clay: A case study[J]. Tunnelling and Underground Space Technology, 2016,60:151-164.

9. PECK R B. Deep excavations and tunneling in soft ground $[\mathrm{C}]$. Proceedings of 7 th International Conference on Soil Mechanics and Foundation Engineering, Mexico City: State of the Art Report, 1969: 225-290.

10. HAN Xuan, LI Ning. Comparative analysis of strata prediction models for ground movement induced by tunnel construction[J]. Journal of Rock Mechanics and Engineering, 2007,03:594-600.

11. Lee K M, Rowe R K, Lo K Y. Subsidence owing to tunneling $\mathrm{I}$ : Estimating the gap parameter[J].Canadian Geotechnical 